\title{
Investigation of Serum Oxidized Low-Density Lipoprotein IgG Levels in Patients with Angiographically Defined Coronary Artery Disease
}

\author{
Mohsen Moohebati, ${ }^{1,2}$ Vahid Kabirirad, ${ }^{3}$ Majid Ghayour-Mobarhan, ${ }^{1,4}$ \\ Habibollah Esmaily, ${ }^{5}$ Shima Tavallaie, ${ }^{1,4}$ Amir Akhavan Rezayat, ${ }^{3}$ \\ Hossein Pourghadamyari, ${ }^{6}$ and Amirhossein Sahebkar ${ }^{7}$ \\ ${ }^{1}$ Cardiovascular Research Center, Mashhad University of Medical Sciences, Mashhad, Iran \\ ${ }^{2}$ Department of Cardiology, Mashhad University of Medical Sciences, Mashhad, Iran \\ ${ }^{3}$ Student Research Committee, Biochemistry of Nutrition Research Center, School of Medicine, \\ Mashhad University of Medical Sciences, Mashhad, Iran \\ ${ }^{4}$ Biochemistry of Nutrition Research Center, School of Medicine, Mashhad University of Medical Sciences, \\ Mashhad 9177948564, Iran \\ ${ }^{5}$ Health Sciences Research Center, Department of Biostatistics and Epidemiology, School of Health, \\ Mashhad University of Medical Sciences, Mashhad, Iran \\ ${ }^{6}$ Department of Clinical Biochemistry, Tehran University of Medical Sciences, Tehran, Iran \\ ${ }^{7}$ Biotechnology Research Center, Mashhad University of Medical Sciences, Mashhad, Iran
}

Correspondence should be addressed to Majid Ghayour-Mobarhan; ghayourm@mums.ac.ir

Received 14 July 2013; Revised 22 September 2013; Accepted 6 October 2013; Published 3 February 2014

Academic Editor: Karlheinz Peter

Copyright (C) 2014 Mohsen Moohebati et al. This is an open access article distributed under the Creative Commons Attribution License, which permits unrestricted use, distribution, and reproduction in any medium, provided the original work is properly cited.

It has been suggested that antioxidized low-density lipoprotein (anti-oxLDL) antibodies play a role in the pathogenesis of atherosclerosis. The aim of this study was to measure serum ox-LDL IgG levels in 31 patients with angiographically defined coronary artery disease (CAD) ( $\geq 50 \%$ stenosis in at least one major coronary artery; $\mathrm{CAD}^{+}$group) and compare these levels with those of 32 subjects with $<50 \%$ coronary stenosis ( $\mathrm{CAD}^{-}$group) and 24 healthy age- and sex-matched controls using ELISA. We did not find any significant difference between $\mathrm{CAD}^{+}, \mathrm{CAD}^{-}$, and control groups in regard to oxLDL IgG levels $(P=0.83)$. Serum oxLDL IgG levels did not differ between 1VD (one vessel disease), 2VD (2 vessels disease), and 3VD (3 vessels disease) subgroups of CAD ${ }^{+}$ patients $(P=0.20)$. Serum anti-oxLDL titers were only significantly correlated with LDL-C in the $\mathrm{CAD}^{+}$group $(P<0.05)$ and waist and hip circumference $\left(P<0.05\right.$ and $P<0.01$, resp.) in the CAD ${ }^{-}$group. In stepwise regression analysis, none of the conventional cardiovascular risk factors was associated with serum ox-LDL IgG levels. The present results suggest that serum levels of ox-LDL IgG are neither associated with the presence and severity of CAD nor with the conventional cardiovascular risk factors.

\section{Introduction}

Coronary artery disease (CAD) is a major cause of mortality and disability worldwide [1]. The major independent risk factors for CAD including age, gender, hypertension, cigarette smoking, diabetes mellitus, elevated serum levels of total and low-density lipoprotein cholesterol (LDL-C), and diminished levels of high-density lipoprotein cholesterol
(HDL-C) are usually applied for the assessment and management of cardiovascular risk [2]. However, these established risk factors can only explain about $25-30 \%$ of the total cardiovascular risk in patients [3], suggesting that other potential factors also play an important role in the pathogenesis of atherosclerosis and CAD. In recent decades, oxidative stress [4], inflammation [5], and immune responses [6] have been considered as emerging risk factors that 
can significantly contribute to the development of vascular events.

Oxidized low-density lipoproteins (ox-LDLs) are believed to play a pivotal role in atherogenesis [7]. Oxidative modification of LDL is a prerequisite for the accumulation of LDL in macrophages and formation of foam cells. Physicochemical and immunological properties of LDL particles isolated from atherosclerotic lesions have been found to resemble those of ox-LDL [8]. Moreover, immunocytochemical investigations have identified both ox-LDL epitopes and anti-ox-LDL immunoglobulins within atherosclerotic lesions [8-10]. Notably, antioxidant therapy has been shown to reduce atherogenesis in experimental animal models [11, 12]. Antibodies against malondialdehyde- (MDA-) modified LDL have been reported to predict the progression of carotid atherosclerosis [13], CAD [14], and myocardial infarction [15]. Furthermore, results from studies by Heitzer et al. [16] and Raitakari et al. [17] have indicated that antibodies against $\mathrm{Cu}^{2+}$-oxidized LDL are correlated with endothelial dysfunction.

Although immune response against ox-LDL has been suggested by some studies to be associated with the severity of atherosclerosis $[18,19]$, there has been little data examining the relationship between ox-LDL IgG levels and CAD based on angiography assessment. Therefore, we principally aimed to determine whether IgG antibodies against ox-LDL are associated with CAD. Since conventional cardiovascular risk factors may influence lipid metabolism and immune function, bivariate correlations between serum ox-LDL IgG levels and CAD risk factors and also determinants of anti-oxLDL levels were sought as ancillary aims of the present study.

\section{Methods}

2.1. Study Population. The study population consisted of 63 patients (27 females, 34 males) who were selected from subjects undergoing coronary angiography in the Ghaem Hospital (Mashhad, Iran). Indication of angiography in these patients was for stable angina based on presence of myocardial ischemia in at least one of the following objective tests: exercise test, thallium single photon emission computed tomography (SPECT), and dobutamin stress echocardiography. Coronary angiography was performed using routine procedures. Analysis of the angiograms was performed offline by a specialist cardiologist. The presence of one or more stenoses $\geq 50 \%$ in diameter of at least one major coronary artery (left main, right, left anterior descending, or circumflex artery) was considered as evidence of significant CAD $[20,21]$. Patients with stenoses of $\leq 50 \%$ in all major coronary arteries were considered to have a normal angiogram $\left(\mathrm{CAD}^{-}\right)$.

The $\mathrm{CAD}^{+}$patients (i.e., those with at least $\geq 50 \%$ stenosis in at least one major coronary artery) were classified according to the number of significantly affected stenotic vessels into single vessel (1VD; $n=7), 2$ vessels (2VD; $n=13$ ), and 3 vessels ( $3 \mathrm{VD} ; n=11$ ) disease subgroups. Selected $\mathrm{CAD}^{+}(n=31 ; 12$ females, 19 males; mean age: $59.39 \pm 10.14$ years $)$ and $\mathrm{CAD}^{-}(n=32 ; 15$ females, 17 males; mean age:
$58.34 \pm 9.68$ years) patients were matched for age and gender. Age- and sex-matched healthy volunteers were also recruited as a normal control group ( $n=24 ; 6$ females, 18 males; mean age: $58.25 \pm 9.19$ years). The control subjects had never experienced any symptom nor had any signs of CAD. These subjects had no other apparent major disease. Information on smoking, drug use, and family history of CAD was obtained via a questionnaire.

2.2. Anthropometric and Other Measurements. For all patients, anthropometric parameters including weight, height, and body mass index (BMI) were measured. Weight was measured with the subjects dressed in light clothing after an overnight fasting using a standard scale. Blood pressure was measured twice while the patients were seated and rested, using a standard mercury sphygmomanometer. The systolic blood pressure was defined as the appearance of the first sound (Korotkoff phase 1), and the diastolic blood pressure was defined as the disappearance of the sound (Korotkoff phase 5) during deflating of the cuff. BMI was calculated as weight (in kilograms) divided by height squared (in square meters).

2.3. Blood Sampling and Routine Biochemical Analysis. Blood samples were taken from patients prior to angiography procedure. Following venepuncture, blood samples were collected into Vacutainer tubes and centrifuged at 10,000 $\times \mathrm{g}$ for $15 \mathrm{~min}$ at $4^{\circ} \mathrm{C}$. After separation, aliquots of serum were frozen at $-80^{\circ} \mathrm{C}$ until analysis.

2.4. Routine Biochemical Analysis. A full-fasted lipid profile was determined for each subject. Serum lipid and fasting blood glucose (FBS) concentrations were measured by enzymatic methods.

2.5. Antioxidized LDL Antibody Assay. IgG autoantibodies against ox-LDL were assayed by ELISA using a commercially available ELISA kit (ox-LDL Antibody ELISA kit, Immundiagnostik AG, Germany), as described by Marroquin et al. [20] and according to manufacturer's specifications. This kit uses individual microplate strips that were coated with native or ox-LDL. The antigen stability was shown to last for at least 4 months. Sera were diluted 1:101 prior to the assay.

2.6. Statistical Analysis. All statistical analyses were performed using the SPSS for Windows, version 11.5 (SPSS Inc., Chicago, IL, USA). Data were expressed as mean \pm SD or median and interquartile range (in case of ox-LDL IgG levels). Group comparisons were performed using one-way ANOVA. Categorical data were compared using Chi-square test. A two-sided $P$ value of $<0.05$ was considered as statistically significant. Bivariate correlations between different parameters and ox-LDL IgG levels were assessed using Pearson's and Spearman's rank correlation coefficients for normally and nonnormally distributed data, respectively. Stepwise multiple linear regression analysis was used to identify determinants of ox-LDL IgG levels. Ox-LDL IgG levels were entered into the model after a square root transformation. Dichotomous 
$(1=$ yes $/ 0=$ no $)$ variables that were entered into the model included diabetes mellitus, hyperlipidemia, hypertension, and smoking. Height, weight, FBS, waist circumference, hip circumference, high-density lipoprotein (HDL), systolic blood pressure, and number of narrowed vessels (VD) were entered as continuous variables in the same model. Data for age and gender were not included since the patients and healthy subjects were matched for these parameters.

\section{Results}

3.1. Demographic Characteristics. The three study groups $\left(\mathrm{CAD}^{+}, \mathrm{CAD}^{-}\right.$, and control) were matched for age and gender. There was no significant difference in BMI and waist/hip ratio between the groups $(P>0.05)$. However, height, waist circumference, and hip circumference in both $\mathrm{CAD}^{+}(P<0.05, P<0.05$, and $P<0.01$, resp. $)$ and $\mathrm{CAD}^{-}$ $(P<0.01, P<0.05$, and $P<0.01$, resp. $)$ groups were significantly lower than those in the control group. Weight was significantly lower in the $\mathrm{CAD}^{-}$versus control group $(P<0.01)$. Mean systolic blood pressure was significantly higher in both $\mathrm{CAD}^{+}$and $\mathrm{CAD}^{-}$groups compared with the control group $(P<0.001)$. No significant difference was observed in diastolic blood pressure, FBS, and CRP between the three groups $(P>0.05)$. Likewise, no significant difference in lipid profile parameters (HDL-C, LDL-C, and triglycerides) was observed among the three groups $(P>$ 0.05, Table 1). Demographic parameters were comparable between subgroups of $\mathrm{CAD}^{+}$patients in terms of the number of stenosed vessels (1VD, 2VD, and 3VD) $(P>0.05$, Table 2). Demographic characteristics of study groups are summarized in Tables 1 and 2.

3.2. Ox-LDL IgG Levels in relation to CAD. There was no significant difference between $\mathrm{CAD}^{+}, \mathrm{CAD}^{-}$, and control groups regarding anti-ox-LDL concentrations $(P=0.83$, Figure 1). With respect to the severity of CAD, there was no significant difference in ox-LDL IgG levels between $1 \mathrm{VD}$, 2 VD, and 3 VD subgroups $(P=0.20$, Figure 2$)$.

\subsection{Correlations between Serum Ox-LDL IgG Levels and CVD}

Risk Factors. In bivariate analyses, serum ox-LDL IgG levels were only significantly correlated with waist circumference and hip circumference $(P<0.05$ and $P<0.01$, resp.) in the $\mathrm{CAD}^{-}$group, and LDL-C $(P<0.05)$ in the $\mathrm{CAD}^{+}$group. In the control group, no significant correlation was found between serum ox-LDL IgG levels and CVD risk factors $(P>$ 0.05 , Table 3 and Figure 3).

3.4. Association between Serum Ox-LDL IgG Levels and CVD Risk Factors. In stepwise multiple linear regression analysis, none of CVD risk factors had a significant independent association with serum ox-LDL IgG levels (Table 4).

\section{Discussion}

The present study set out to determine serum ox-LDL IgG levels in $\mathrm{CAD}^{+}$patients with significant $(\geq 50 \%$ in luminal

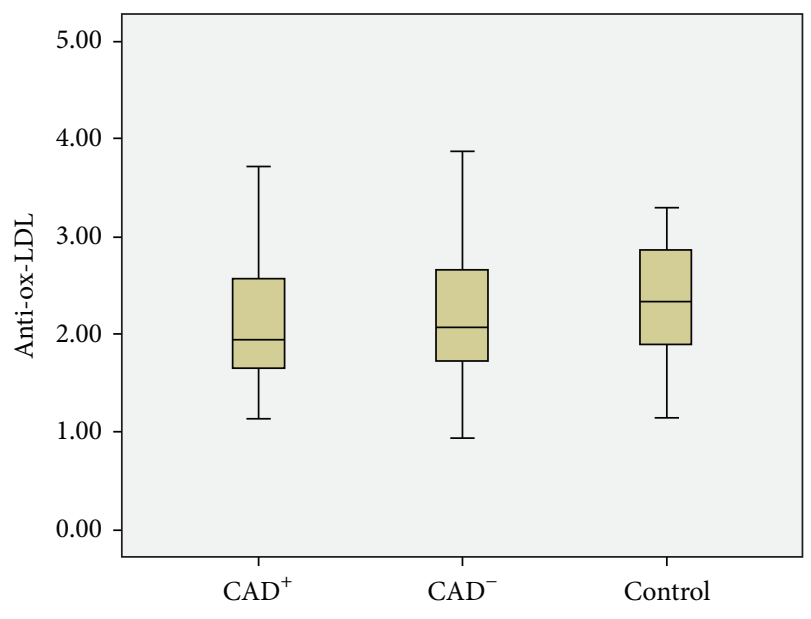

FIGURE 1: Boxplot of anti-ox-LDL concentrations in $\mathrm{CAD}^{+}, \mathrm{CAD}^{-}$, and control groups. No significant difference was observed among the three groups $(P>0.05)$.

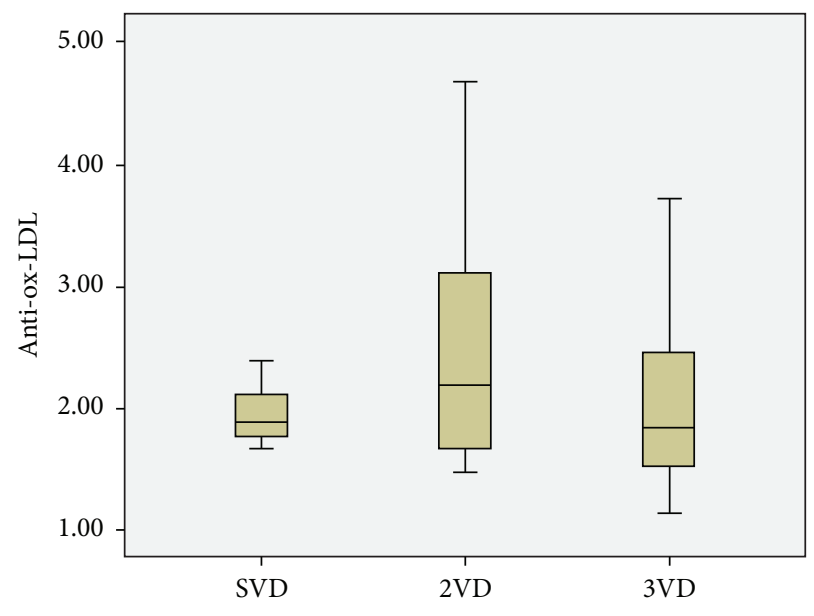

FIgURE 2: Boxplot of anti-ox-LDL concentration in SVD, 2VD, and $3 \mathrm{VD}$ subgroups of $\mathrm{CAD}^{+}$patients. No significant difference was observed among the three groups.

diameter) coronary stenosis compared to subjects who are either normal or have stenoses $<50 \%$. As a secondary aim, associations between anti-ox-LDL IgG levels and CAD risk factors were explored. The findings did not reveal any significant difference between $\mathrm{CAD}^{+}, \mathrm{CAD}^{-}$, and control groups regarding serum ox-LDL IgG levels. Likewise, there was no association between antibody levels and severity of $\mathrm{CAD}$ based on the number of stenosed vessels. In consistence, serum anti-ox-LDL IgG levels were found to be independent of $\mathrm{CAD}$ risk factors. Heretofore, several clinical studies have been published on the role of ox-LDL IgG levels in the initiation or progression of CVD. However, data have been controversial. Erkkilä et al. [22] measured levels of autoantibodies against ox-LDL in 415 patients with different manifestations of coronary heart disease (CHD). Anti-oxLDL antibodies were significantly higher in the group with acute myocardial infarction (AMI) compared to other groups 
TABLE 1: Demographic and clinical characteristics of $\mathrm{CAD}^{+}, \mathrm{CAD}^{-}$, and control subjects.

\begin{tabular}{|c|c|c|c|}
\hline & $\mathrm{CAD}^{+}$ & $\mathrm{CAD}^{-}$ & Control \\
\hline Number of subjects & 31 & 32 & 24 \\
\hline Gender (F/M) & $19 / 12^{\mathrm{f}}$ & $17 / 15$ & $6 / 18$ \\
\hline Age & $59.39 \pm 10.14$ & $58.34 \pm 9.68$ & $58.25 \pm 9.19$ \\
\hline Height & $159.07 \pm 8.54^{\mathrm{a}}$ & $157.83 \pm 11.99^{\mathrm{b}}$ & $167.10 \pm 7.77$ \\
\hline Weight & $69.71 \pm 11.93$ & $64.40 \pm 14.48^{\mathrm{b}}$ & $75.74 \pm 12.67$ \\
\hline FBS & $138.07 \pm 81.75$ & $99.14 \pm 28.34$ & $94.18 \pm 16.62$ \\
\hline BMI & $27.39 \pm 3.63$ & $26.05 \pm 6.87$ & $27.60 \pm 4.28$ \\
\hline Waist/hip ratio & $0.94 \pm 0.08$ & $0.94 \pm 0.07$ & $0.95 \pm 0.16$ \\
\hline Waist circumference & $88.28 \pm 9.54^{\mathrm{a}}$ & $88.77 \pm 13.55^{\mathrm{a}}$ & $99.16 \pm 19.01$ \\
\hline Hip circumference & $93.88 \pm 8.39^{\mathrm{b}}$ & $94.12 \pm 12.44^{\mathrm{b}}$ & $105.32 \pm 15.86$ \\
\hline LDL-C & $90.67 \pm 31.84$ & $107.57 \pm 46.99$ & $106.78 \pm 20.17$ \\
\hline HDL-C & $40.60 \pm 15.91$ & $38.88 \pm 10.88$ & $64.70 \pm 88.93$ \\
\hline TG & $123.69(47.00-331.00)$ & $157.00(41.00-352.00)$ & $146.65(53.00-325.00)$ \\
\hline SBP & $156.45 \pm 30.30^{c}$ & $154.08 \pm 28.18^{\mathrm{c}}$ & $123.92 \pm 10.59$ \\
\hline DBP & $77.87 \pm 14.47$ & $76.88 \pm 14.88$ & $80.35 \pm 8.87$ \\
\hline Diabetes mellitus (\%) & $41.9^{\mathrm{f}}$ & 7.7 & 12.5 \\
\hline Smoking or addiction (\%) & 51.6 & 35.5 & 12.5 \\
\hline Hypertension (\%) & 64.5 & 46.2 & 41.7 \\
\hline Hyperlipidemia (\%) & 51.6 & 38.5 & 22.2 \\
\hline
\end{tabular}

Values are presented as mean \pm SD. FBS: fasting blood sugar; BMI: body mass index; LDL-C: low-density lipoprotein cholesterol; HDL-C: high-density lipoprotein cholesterol; TG: triglycerides; SBP: systolic blood pressure; DBP: diastolic blood pressure. Compared with the control group: ${ }^{\mathrm{a}} P<0.05,{ }^{\mathrm{b}} P<0.01$, and ${ }^{\mathrm{c}} P<0.001$; compared with the $\mathrm{CAD}^{-}$group: ${ }^{\mathrm{d}} P<0.01$, ${ }^{\mathrm{e}} P<0.001$; comparison between all groups (using Chi-square test): ${ }^{\mathrm{f}} P<0.05,{ }^{\mathrm{g}} P<0.001$.

TABLE 2: Demographic and clinical characteristics of $\mathrm{CAD}^{+}$subjects with 1, 2, and 3 narrowed vessels.

\begin{tabular}{lccc}
\hline & SVD & 2 VD & 3VD \\
\hline Number of subjects & 7 & 13 & $5 / 6$ \\
Gender (F/M) & $6 / 1$ & $8 / 5$ & $58.27 \pm 12.37$ \\
Age & $62.14 \pm 11.09$ & $58.85 \pm 7.90$ & $159.54 \pm 6.84$ \\
Height & $160.00 \pm 7.70$ & $158.38 \pm 10.46$ & $67.40 \pm 13.26$ \\
Weight & $75.12 \pm 7.66$ & $70.00 \pm 12.01$ & $120.60 \pm 70.98$ \\
FBS & $167.42 \pm 84.27$ & $135.27 \pm 91.29$ & $26.60 \pm 4.00$ \\
BMI & $29.07 \pm 0.89$ & $27.63 \pm 3.76$ & $0.91 \pm 0.05$ \\
Waist/hip ratio & $1.01 \pm 0.13$ & $0.93 \pm 0.07$ & $83.90 \pm 7.14$ \\
Waist circumference & $94.00 \pm 6.21$ & $90.18 \pm 11.17$ & $91.60 \pm 8.72$ \\
Hip circumference & $94 \pm 12.72$ & $95.90 \pm 6.51$ & $88.70 \pm 29.34$ \\
LDL-C & $94.92 \pm 17.45$ & $90.23 \pm 43.19$ & $36.63 \pm 14.42$ \\
HDL-C & $50.40 \pm 20.16$ & $38.95 \pm 14.04$ & $125.22(60.00-207.00)$ \\
TG & $89(47.00-128.00)$ & $141.44(64.00-331.00)$ & $146.36 \pm 33.24$ \\
SBP & $154.28 \pm 27.75$ & $166.15 \pm 28.14$ & $73.63 \pm 11.20$ \\
DBP & $74.14 \pm 13.90$ & $83.46 \pm 16.25$ & 36.4 \\
Diabetes mellitus (\%) & 57.1 & 38.5 & 63.6 \\
Smoking or addiction (\%) & 57.1 & 38.5 & 72.7 \\
Hypertension (\%) & 71.4 & 53.8 & 53.8 \\
Hyperlipidemia (\%) & 42.9 & 54.5 & \\
\hline
\end{tabular}

Values are presented as mean \pm SD. FBS: fasting blood sugar; BMI: body mass index; LDL-C: low-density lipoprotein cholesterol; HDL-C: high-density lipoprotein cholesterol; TG: triglycerides; SBP: systolic blood pressure; DBP: diastolic blood pressure. No significant difference between three groups $(P>0.05)$. 
TABLE 3: Correlations between serum oxLDL IgG levels and CVD risk factors.

\begin{tabular}{|c|c|c|c|c|c|c|}
\hline & \multicolumn{2}{|c|}{$\mathrm{CAD}^{+}$} & \multicolumn{2}{|c|}{$\mathrm{CAD}^{-}$} & \multicolumn{2}{|c|}{ Control } \\
\hline & $r$ values & $P$ values & $r$ values & $P$ values & $r$ values & $P$ values \\
\hline Age* $^{*}$ & -0.11 & 0.52 & 0.15 & 0.40 & -0.02 & 0.90 \\
\hline Height* & -0.27 & 0.15 & 0.15 & 0.41 & 0.04 & 0.84 \\
\hline Weight* & -0.12 & 0.53 & 0.24 & 0.19 & 0.07 & 0.74 \\
\hline $\mathrm{FBS}^{* *}$ & 0.25 & 0.19 & 0.02 & 0.92 & -0.11 & 0.62 \\
\hline BMI* $^{*}$ & 0.08 & 0.68 & 0.02 & 0.90 & 0.18 & 0.38 \\
\hline Waist/hip ratio* & -0.14 & 0.47 & 0.06 & 0.73 & -0.18 & 0.37 \\
\hline Waist circumference ${ }^{*}$ & 0.06 & 0.77 & 0.41 & 0.02 & -0.16 & 0.43 \\
\hline Hip circumference* & 0.20 & 0.32 & 0.45 & 0.009 & 0.06 & 0.75 \\
\hline $\mathrm{LDL}^{* *}$ & 0.46 & 0.03 & 0.06 & 0.77 & -0.30 & 0.21 \\
\hline $\mathrm{HDL}^{* *}$ & 0.10 & 0.65 & -0.30 & 0.16 & -0.29 & 0.22 \\
\hline $\mathrm{TG}^{*}$ & -0.02 & 0.91 & 0.16 & 0.43 & 0.007 & 0.97 \\
\hline $\mathrm{SBP}^{*}$ & 0.12 & 0.49 & 0.13 & 0.50 & 0.05 & 0.84 \\
\hline $\mathrm{DBP}^{*}$ & 0.14 & 0.43 & 0.26 & 0.19 & 0.27 & 0.34 \\
\hline
\end{tabular}

${ }^{*}$ Pearson's correlation analysis.

${ }^{* *}$ Spearman's correlation analysis.

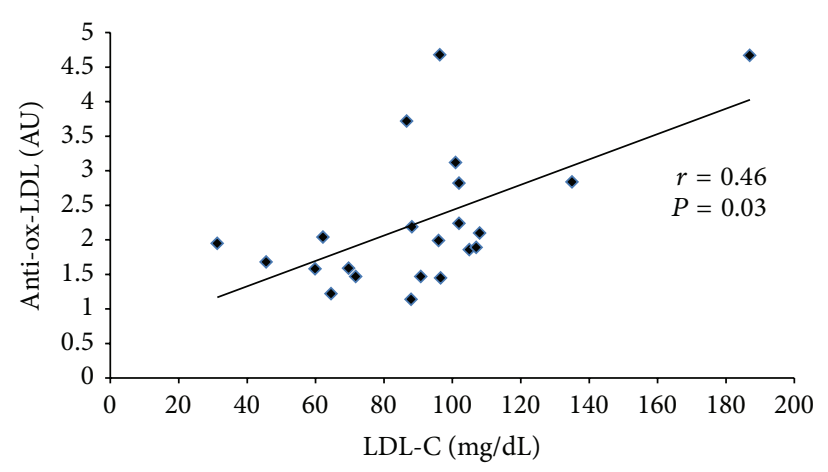

(a)

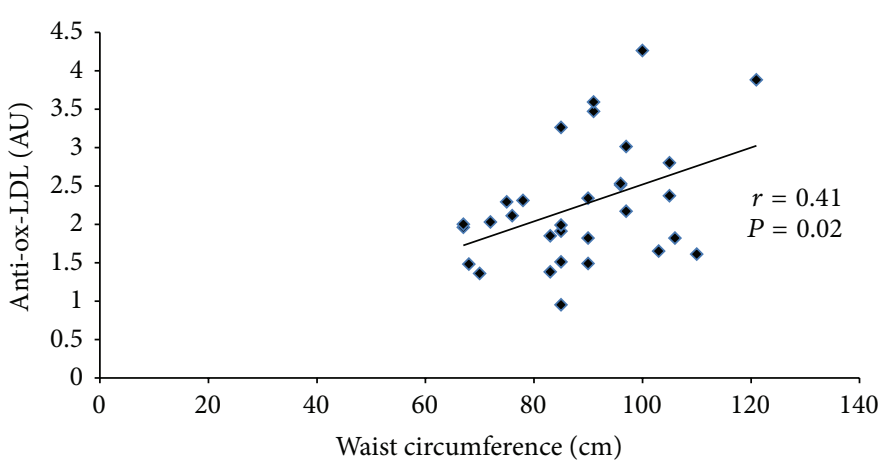

(b)

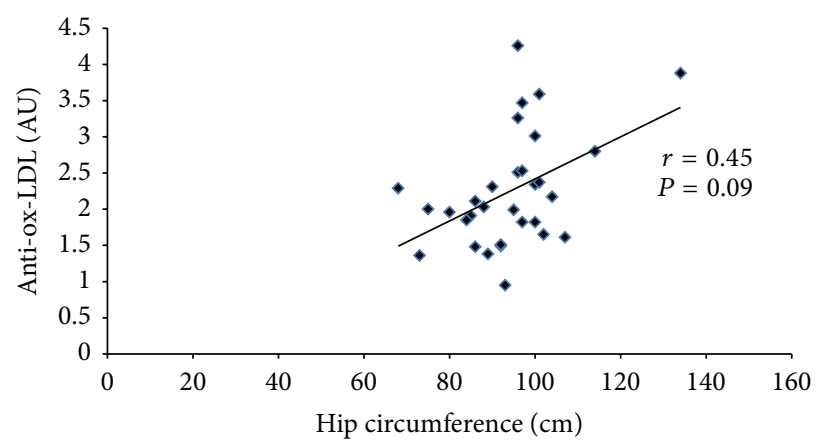

(c)

FIGURE 3: Scatter plots depicting significant correlations between serum ox-LDL IgG levels and (a) LDL-C (in the CAD ${ }^{+}$group), (b) waist circumference (in the $\mathrm{CAD}^{-}$group), and (c) hip circumference (in the $\mathrm{CAD}^{-}$group). AU: absorbance unit.

with coronary artery bypass surgery, balloon angioplasty, and acute myocardial ischemia in men, but not in women. Therefore, it was concluded that autoantibodies against oxLDL are associated with AMI in men. Lehtimäki et al. [23] determined serum levels of antibodies against $\mathrm{Cu}^{2+}$ oxidized LDL in 58 patients with angiographically verified
CAD and 34 controls without CAD and concluded that elevated levels of anti-ox-LDL are associated with CAD. Inoue et al. [24] measured ox-LDL IgG levels in 108 patients who had angiographically verified $\mathrm{CAD}$ and 31 patients who had chest pain but no significant CAD as controls. The oxLDL antibody levels were found to be higher in patients 
TABLE 4: Association between serum anti-oxLDL titres and CVD risk factors in stepwise multiple regression analysis.

\begin{tabular}{lccc}
\hline Covariate & $\beta$ & $P$ & $95 \%$ confidence interval \\
\hline Age & -.162 & .616 & $-.096-0.60$ \\
FBS & .402 & .290 & $-.007-0.020$ \\
LDL-C & .090 & .759 & $-.021-0.028$ \\
HDL-C & -.290 & .349 & $-.085-0.034$ \\
TG & .005 & .990 & $-.016-0.016$ \\
SBP & .136 & .693 & $-.022-0.031$ \\
DBP & -.388 & .389 & $-.099-0.043$ \\
DM & .227 & .546 & $-1.676-2.938$ \\
Smoking & .105 & .699 & $-.482-0.684$ \\
HTN & .360 & .275 & $-.720-2.210$ \\
HLP & -.123 & .592 & $-1.337-0.815$ \\
BMI & -.651 & .615 & $-.599-0.378$ \\
Waist hip ratio & -2.085 & .430 & $-105.209-49.503$ \\
\hline
\end{tabular}

FBS: fasting blood sugar; BMI: body mass index; LDL-C: low-density lipoprotein cholesterol; HDL-C: high-density lipoprotein cholesterol; TG: triglycerides; SBP: systolic blood pressure; DBP: diastolic blood pressure; HTN: hypertension; HLP: hyperlipidemia; BMI: body mass index.

with multivessel CAD compared to controls. The levels were also higher in patients with unstable angina or in patients with AMI compared to patients with stable-effort angina or old MI. Laczik et al. [25] measured serum ox-LDL IgG levels in a total of 54 patients with acute coronary syndrome (ACS) and 41 matched healthy controls in a prospective study. Higher levels were found in patients with ACS versus controls and it was suggested that such raised levels might have a role in plaque destabilization. Tsimikas et al. [26] investigated the relationship between IgG autoantibodies to ox-LDL and CAD and cardiovascular events in 504 patients undergoing clinically indicated coronary angiography. The results indicated that anti-ox-LDL levels are positively associated with the presence of angiographically determined $\mathrm{CAD}$. Along with the aforementioned studies, there are other reports that support the present findings on the lack of association between anti-ox-LDL levels and CAD: Rossi et al. [27] measured the levels of ox-LDL IgG antibodies in 529 consecutive patients undergoing quantitative coronary angiography for suspected CAD. They found no significant association between anti-ox-LDL levels and CAD severity. Consistently, Virella et al. [28] showed that anti-ox-LDL levels are neither associated with CAD severity nor plasma lipid levels in healthy individuals. Chen et al. [29] examined the association of ox-LDL antibody levels with the severity of CAD in 558 subjects from the Women's Ischemia Syndrome Evaluation Study. Their findings implied that higher ox-LDL IgM levels may provide protection against coronary stenosis. This study is in agreement with that of Rossi et al. [27] who reported an inverse association between ox-LDL IgM levels and presence of angiographically determined CAD. Finally, Che et al. [30] measured the levels of IgG anti-ox-LDL in 154 patients undergoing coronary angiography for suspected $\mathrm{CHD}$ and reported that such levels are lower in CHD patients compared to controls.

Taken collectively, it appears that circulating ox-LDL IgG levels are elevated following an acute coronary event, for example, episodes of AMI. This might be due to the impact of plaque rupture which leads to the release and exposure of considerable plaque content of ox-LDL to the circulation, thereby stimulating immune system to produce humoral response. The inconsistent findings on the association of oxLDL antibody levels with CAD could be attributed to the fact that coronary angiography focuses on the arterial lumen rather than the arterial wall, whilst it has been suggested that rupture-prone (vulnerable) plaques are not those that yield a high degree of stenosis [27]. Moreover, antibodies against ox-LDL have been shown to be heterogenous and IgG and IgM subclasses are known to have different profile of changes following $\mathrm{CAD}$, suggesting another reason for discrepant findings on the anti-ox-LDL status in CAD patients. A number of previous reports which reported elevation of antiox-LDL levels following CAD are subject to selection bias $[24,31]$. On the other hand a previous large-scale study has provided results very similar to those in the present study [27]. Finally, antibody levels might be affected by ethnicity and a potential explanation for the discrepant findings might be that the profile of immunoglobulin changes differs among ethnicities.

A number of limitations must be acknowledged for the present study. First, only IgG levels against ox-LDL were determined. Besides, the IgGs against ox-LDL are themselves a mixture of antibodies with different specificities, whilst it has been shown that only IgG subclasses against certain epitopes of ox-LDL are implicated in atherogenesis [32]. Second, this study was exclusively conducted among Caucasian subjects and thus was not able to look at a plausible impact of ethnicity on ox-LDL IgG levels. Hence, the findings may not be attributable to CAD patients from author descents. Third, coronary angiography may not be an ideal indicator of arterial wall pathology and rupture-prone atherosclerotic plaques that are more likely to contain significant ox-LDL content.

Given the above limitations, future studies are warranted to assess both IgG and IgM levels against ox-LDL and also quantify different IgG subclasses based on their affinity to 
ox-LDL. In addition, quantification of ox-LDL antibodies is encouraged to be conducted in patients with acute coronary syndrome because these patients have vulnerable plaques that are known to contain higher amounts of ox-LDL and corresponding antibodies.

It is also recommended to further explore the association between ox-LDL IgG levels and surrogate markers of CAD including carotid intima-media thickness and brachial artery flow-mediated dilation. Finally, any predictive value of oxLDL IgG levels for CVD endpoints needs to be investigated by prospective studies.

\section{Conclusions}

In summary, findings arising from the present study indicated the lack of clinically significant association between circulating ox-LDL IgG levels, and the presence, severity and conventional risk factors of CAD. With the current available evidence, ox-LDL IgG levels cannot be used for the risk assessment of CAD.

\section{Conflict of Interests}

The authors declare that they have no conflict of interests.

\section{Authors' Contribution}

Mohsen Mouhebati and Vahid Kabirirad contributed equally to this work.

\section{Acknowledgments}

The authors are particularly grateful to the patients and their family members who volunteered to participate in this study. This work was financially supported by the Mashhad University of Medical Sciences, Mashhad, Iran. This article was extracted from the thesis prepared by Vahid Kabirirad to fulfill the requirements for earning the MD degree from the Mashhad University of Medical Sciences (thesis no. 6755).

\section{References}

[1] D. Lloyd-Jones, R. J. Adams, T. M. Brown et al., "Executive summary: heart disease and stroke statistics-2010 update: a report from the American Heart Association," Circulation, vol. 121, pp. 948-954, 2010.

[2] P. W. F. Wilson, R. B. D’Agostino, D. Levy, A. M. Belanger, H. Silbershatz, and W. B. Kannel, "Prediction of coronary heart disease using risk factor categories," Circulation, vol. 97, no. 18, pp. 1837-1847, 1998.

[3] E. L. Bierman, "George Lyman Duff memorial lecture. Atherogenesis in diabetes," Arteriosclerosis and Thrombosis, vol. 12, no. 6, pp. 647-656, 1992.

[4] U. Landmesser, S. Spiekermann, S. Dikalov et al., "Vascular oxidative stress and endothelial dysfunction in patients with chronic heart failure: role of xanthine-oxidase and extracellular superoxide dismutase," Circulation, vol. 106, no. 24, pp. 30733078, 2002.
[5] T. A. Pearson, G. A. Mensah, R. W. Alexander et al., "Markers of inflammation and cardiovascular disease: application to clinical and public health practice: a statement for healthcare professionals from the centers for disease control and prevention and the American heart association," Circulation, vol. 107, no. 3, pp. 499-511, 2003.

[6] M. Ghayour-Mobarhan, A. A. Rahsepar, S. Tavallaie, S. Rahsepar, and G. A. Ferns, "The potential role of heat shock proteins in cardiovascular disease: evidence from in vitro and in vivo studies," Advances in Clinical Chemistry, vol. 48, pp. 27-72, 2009.

[7] S. Parthasarathy, D. Steinberg, and J. L. Witztum, "The role of oxidized low-density lipoproteins in the pathogenesis of atherosclerosis," Annual Review of Medicine, vol. 43, pp. 219225, 1992.

[8] S. Ylä-Herttuala, W. Palinski, S. W. Butler, S. Picard, D. Steinberg, and J. L. Witztum, "Rabbit and human atherosclerotic lesions contain IgG that recognizes epitopes of oxidized LDL," Arteriosclerosis and Thrombosis, vol. 14, no. 1, pp. 32-40, 1994.

[9] W. Palinski, M. E. Rosenfeld, S. Ylä-Herttuala et al., "Low density lipoprotein undergoes oxidative modification in vivo," Proceedings of the National Academy of Sciences of the United States of America, vol. 86, no. 4, pp. 1372-1376, 1989.

[10] S. Ylä-Herttuala, W. Palinski, M. E. Rosenfeld et al., "Evidence for the presence of oxidatively modified low density lipoprotein in atherosclerotic lesions of rabbit and man," Journal of Clinical Investigation, vol. 84, no. 4, pp. 1086-1095, 1989.

[11] T. E. Carew, D. C. Schwenke, and D. Steinberg, "Antiatherogenic effect of probucol unrelated to its hypocholesterolemic effect: evidence that antioxidants in vivo can selectively inhibit low density lipoprotein degradation in macrophage-rich fatty streaks and slow the progression of atherosclerosis in the Watanabe heritable hyperlipidemic rabbit," Proceedings of the National Academy of Sciences of the United States of America, vol. 84, no. 21, pp. 7725-7729, 1987.

[12] T. Kita, Y. Nagano, M. Yokode et al., "Probucol prevents the progression of atherosclerosis in Watanabe heritable hyperlipidemic rabbit, an animal model for familial hypercholesterolemia," Proceedings of the National Academy of Sciences of the United States of America, vol. 84, no. 16, pp. 5928-5931, 1987.

[13] J. T. Salonen, S. Ylä-Herttuala, R. Yamamoto et al., "Autoantibody against oxidised LDL and progression of carotid atherosclerosis," The Lancet, vol. 339, no. 8798, pp. 883-887, 1992.

[14] O. Ben-Yehuda, J. L. Witztum, J. F. Keaney Jr., B. Frei, B. Hankin, and J. A. Vita, "Autoantibody titer to malondialdehyde modified low density lipoprotein correlates with extent of coronary artery disease," Circulation, vol. 94, I638 pages, 1996.

[15] M. Puurunen, M. M. Mänttäri, V. Manninen et al., "Antibody against oxidized low-density lipoprotein predicting myocardial infarction," Archives of Internal Medicine, vol. 154, pp. 26052609, 1994.

[16] T. Heitzer, S. Ylä-Herttuala, J. Luoma et al., "Cigarette smoking potentiates endothelial dysfunction of forearm resistance vessels in patients with Hypercholesterolemia role of oxidized LDL," Circulation, vol. 93, no. 7, pp. 1346-1353, 1996.

[17] O. T. Raitakari, O. P. Pitkänen, T. Lehtimäki et al., "Autoantibody against oxidized LDL and LDL particle size: relationships to coronary reactivity in young men," Journal of the American College of Cardiology, vol. 30, pp. 97-102, 1997. 
[18] M. L. F. Smook, M. van Leeuwen, P. Heeringa et al., "Anti-oxLDL antibody isotype levels, as potential markers for progressive atherosclerosis in APOE and APOECD40L mice," Clinical and Experimental Immunology, vol. 154, no. 2, pp. 264-269, 2008.

[19] M. C. Slot, R. Theunissen, P. van Paassen, J. G. M. C. Damoiseaux, and J. W. Tervaert, "Anti-oxidized low-density lipoprotein antibodies in myeloperoxidase-positive vasculitis patients preferentially recognize hypochlorite-modified low density lipoproteins," Clinical and Experimental Immunology, vol. 149, no. 2, pp. 257-264, 2007.

[20] O. C. Marroquin, K. E. Kip, D. E. Kelley et al., "Metabolic syndrome modifies the cardiovascular risk associated with angiographic coronary artery disease in women: a report from the women's ischemia syndrome evaluation," Circulation, vol. 109, no. 6, pp. 714-721, 2004.

[21] A. Elhendy, J. M. Tsutsui, E. L. O’Leary, F. Xie, A. C. McGrain, and T. R. Porter, "Noninvasive diagnosis of coronary artery disease in patients with diabetes by dobutamine stress real-time myocardial contrast perfusion imaging," Diabetes Care, vol. 28, no. 7, pp. 1662-1667, 2005.

[22] A. T. Erkkilä, O. Närvänen, S. Lehto, M. I. J. Uusitupa, and S. Ylä-Herttuala, "Autoantibodies against oxidized low-density lipoprotein and cardiolipin in patients with coronary heart disease," Arteriosclerosis, Thrombosis, and Vascular Biology, vol. 20, no. 1, pp. 204-209, 2000.

[23] T. Lehtimäki, S. Lehtinen, T. Solakivi et al., "Autoantibodies against oxidized low density lipoprotein in patients with angiographically verified coronary artery disease," Arteriosclerosis, Thrombosis, and Vascular Biology, vol. 19, no. 1, pp. 23-27, 1999.

[24] T. Inoue, T. Uchida, H. Kamishirado, K. Takayanagi, T. Hayashi, and S. Morooka, "Clinical significance of antibody against oxidized low density lipoprotein in patients with atherosclerotic coronary artery disease," Journal of the American College of Cardiology, vol. 37, no. 3, pp. 775-779, 2001.

[25] R. Laczik, P. Szodoray, K. Veres et al., "Assessment of IgG antibodies to oxidized LDL in patients with acute coronary syndrome," Lupus, vol. 20, no. 7, pp. 730-735, 2011.

[26] S. Tsimikas, E. S. Brilakis, R. J. Lennon et al., "Relationship of IgG and IgM autoantibodies to oxidized low density lipoprotein with coronary artery disease and cardiovascular events," Journal of Lipid Research, vol. 48, no. 2, pp. 425-433, 2007.

[27] G. P. Rossi, M. Cesari, R. De Toni et al., "Antibodies to oxidized low-density lipoproteins and angiographically assessed coronary artery disease in white patients," Circulation, vol. 108, no. 20, pp. 2467-2472, 2003.

[28] G. Virella, I. Virella, R. B. Leman, M. B. Pryor, and M. F. LopesVirella, "Anti-oxidized low-density lipoprotein antibodies in patients with coronary heart disease and normal healthy volunteers," International Journal of Clinical \& Laboratory Research, vol. 23, no. 2, pp. 95-101, 1993.

[29] Q. Chen, S. E. Reis, C. Kammerer et al., "Association of antioxidized LDL and candidate genes with severity of coronary stenosis in the women's ischemia syndrome evaluation study," Journal of Lipid Research, vol. 52, no. 4, pp. 801-807, 2011.

[30] J. Che, G. Li, W. Wang et al., "Serum autoantibodies against human oxidized low-density lipoproteins are inversely associated with severity of coronary stenotic lesions calculated by Gensini score," Cardiology Journal, vol. 18, no. 4, pp. 364-370, 2011.
[31] T. Inoue, I. Yaguchi, T. Uchida et al., "Clinical significance of the antibody against oxidized low-density lipoprotein in acute myocardial infarction," Cardiology, vol. 98, no. 1-2, pp. 13-17, 2002.

[32] Á. Zaratin, M. Gidlund, P. Boschcov, L. Castilho, and E. de Faria, "Antibodies against oxidized low-density lipoprotein in normolipidemic smokers," American Journal of Cardiology, vol. 90, no. 6, pp. 651-653, 2002. 


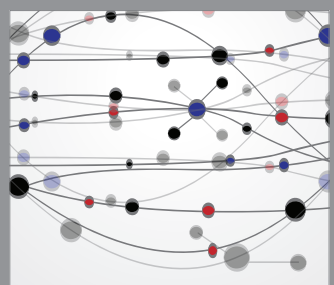

The Scientific World Journal
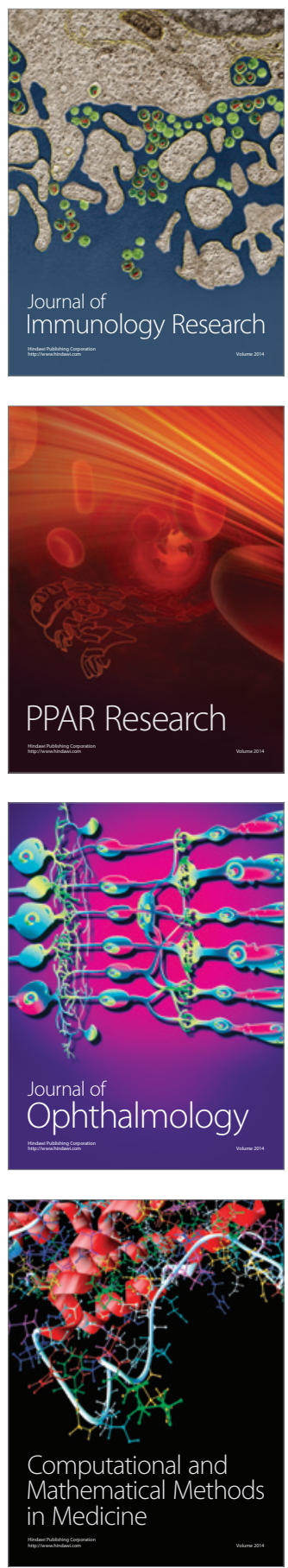

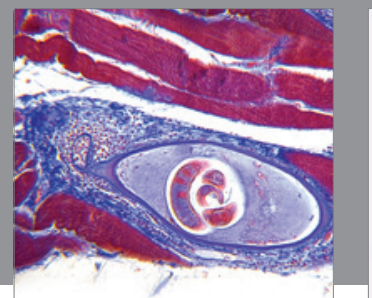

Gastroenterology

Research and Practice
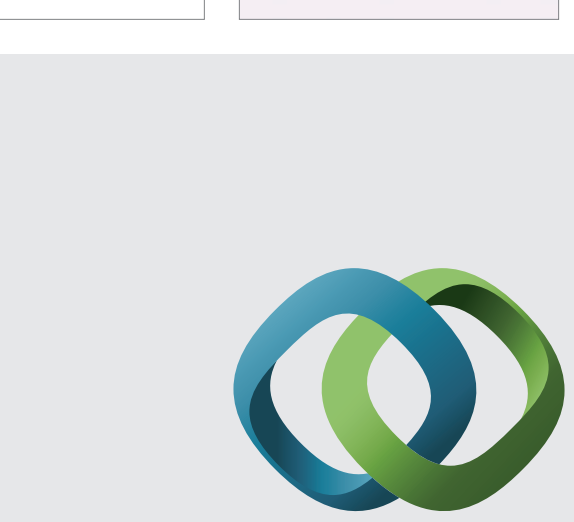

\section{Hindawi}

Submit your manuscripts at

http://www.hindawi.com
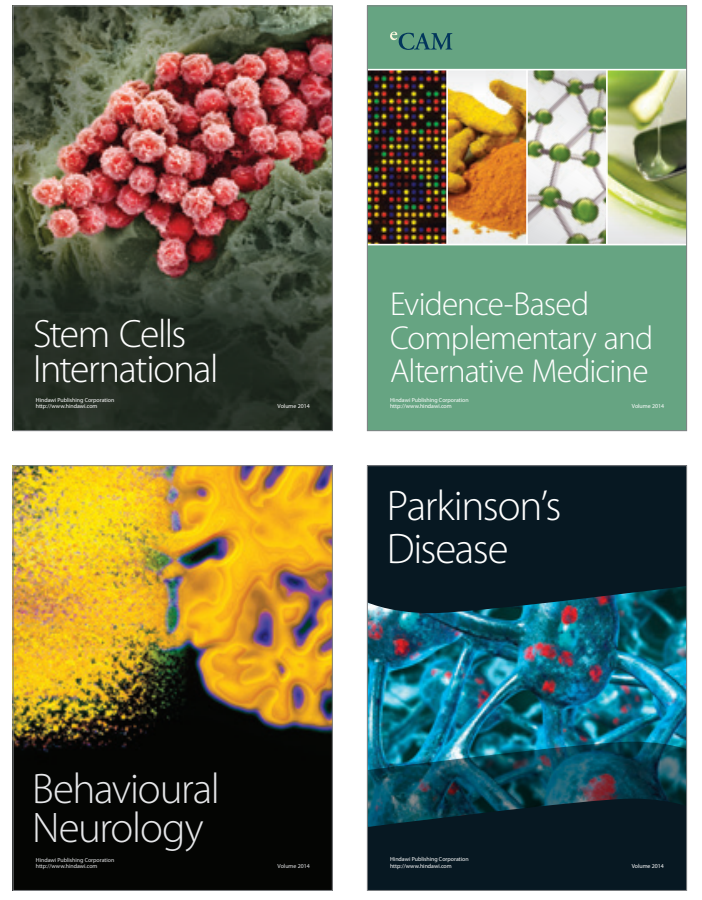
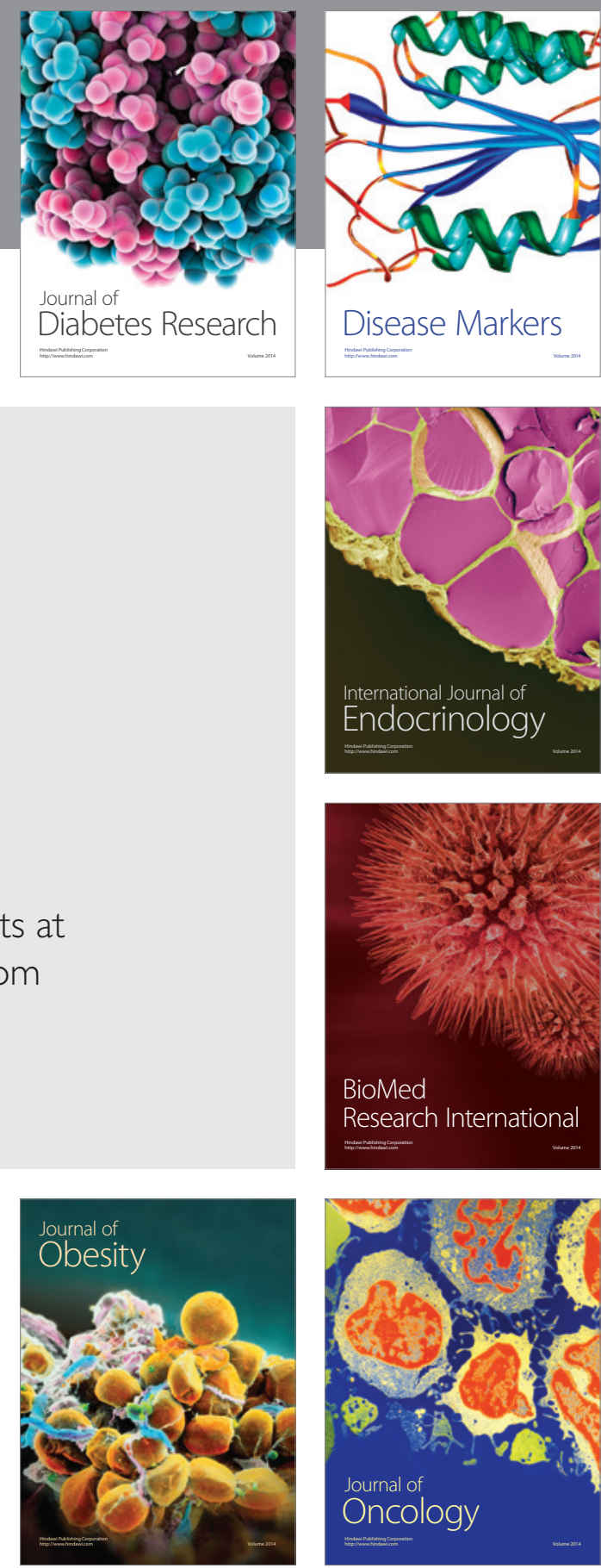

Disease Markers
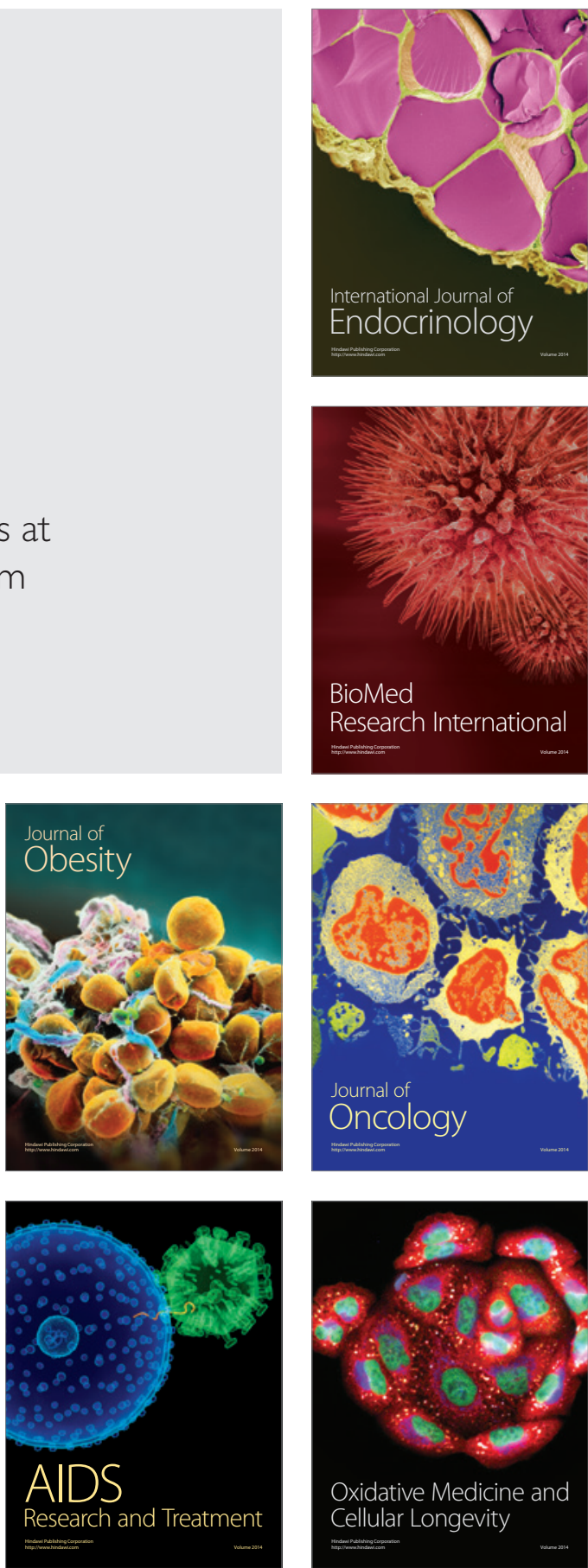